\title{
Délocalisation de diplômes universitaires français au Vietnam dans le cadre du PUF, cas du master Information, Systèmes et Technologie (IST-EEA) de l'université Paris-Sud 11 et l'ENS Cachan
}

\author{
Yves BERNARD, Sylvie RETAILLEAU \\ yves.bernard@supelec.fr, sylvie.retailleau@u-psud.fr \\ LGEP-Supélec, 11 rue Joliot Curie, Plateau de Moulon, 91192 Gif sur Yvette \\ IEF, bâtiment 220, Faculté des Sciences, 91405 Orsay Cedex
}

\begin{abstract}
Résumé : Créé en septembre 2006, le pôle universitaire français (PUF) au Vietnam a pour objectif la délocalisation de diplômes français au Vietnam suivant le schéma européen LMD (licence, master et doctorat). Cet article présente la délocalisation à Hanoï d'une partie du master Information, Systèmes et Technologie (IST-EEA) de l'université ParisSud 11 et l'ENS Cachan. Dans cet article, l'accent est mis sur les retombées de ce type d'échanges pour chaque partenaire. Ce début de troisième année du programme est l'occasion d'un bilan à mi-parcours.
\end{abstract}

Mots clés : international, délocalisation de formations, Vietnam.

\section{INTRODUCTION}

En 2004, la France a signé un accord de coopération universitaire avec le Vietnam. L'université ParisSud 11 et l'Ecole Normale Supérieure (ENS) de Cachan se sont retrouvées impliquées dans cette aventure par le biais du master Information, Systèmes et Technologie (IST-EEA).

La procédure que nous avons suivie n'est pas classique n'ayant pas été associés aux réflexions de ce projet de délocalisation de la formation dès le départ et ayant seulement été impliqués une fois la décision prise. Ainsi nous ne savions pas au départ ce que chaque partenaire aurait à y gagner, ni même si le projet pourrait aboutir. C'est seulement à l'issue des deux premières années que nous pouvons dresser un premier bilan.

L'objectif de cet article n'est donc pas de préciser pourquoi nous avons délocalisé le master IST à Hanoï mais de montrer l'apport de cette délocalisation de formation pour les différents partenaires et notre pédagogie. Avec le recul, il ressort que la solution qui consistait à mettre en place un organe gestionnaire : le PUF (Pôle Universitaire Français) afin de gérer un ensemble de délocalisations était une bonne solution pour permettre la délivrance de diplômes reconnus internationalement au sein des deux universités nationales du Vietnam, notre expérience entrant dans ce cadre.

Nous présentons dans cet article les différentes étapes de la mise en place de la délocalisation de la formation. Les effectifs, correspondant à de petits nombres, sont donnés de manière exhaustive. Nous reviendrons également sur les raisons pour lesquelles les cours sont dispensés le soir et en anglais. Enfin, les clés de la réussite étant liées aux profits que chacun tire de la coopération, nous donnons des exemples d'intérêts individuels. Les conclusions sont présentées sous forme de perspectives pour un projet qui est toujours en cours.

\section{HISTORIQUE DU PROJET}

Le 6 octobre 2004, le Président Jacques Chirac a signé un accord avec le Président vietnamien concer- nant l'enseignement supérieur. Ce protocole d'accord prévoit la mise en place de deux PUF dans les universités nationales du Vietnam à Hanoï (UNVH) et Ho Chi Minh Ville (UNVHCMV). L'objectif figurant dans le protocole initial est triple : 1) Renforcer l'attractivité de l'enseignement supérieur vietnamien. Il faut pour cela proposer des formations conformes aux standards internationaux. 2) Accompagner les universités nationales du Vietnam dans leur mission de «pionnier» en créant des centres d'excellence. 3) Renforcer par une démarche qualité la visibilité des actions menées au titre de la coopération.

Pour créer les formations concernées, le ministère français des affaires étrangères a engagé 3 millions d'euros sur quatre ans. Ce fond devait contribuer au lancement des formations jusqu'à l'atteinte de l'équilibre financier et permettre l'achat de matériel, de documentation et la formation de formateurs vietnamiens. À ce budget vient s'ajouter le montant des frais de scolarité demandés aux étudiants.

Pour ce projet, l'UFR (Unité de Formation et de Recherche) des sciences de l'université Paris-Sud 11 a proposé de délocaliser le master IST, qui est en habilitation partagée avec l'ENS Cachan, et qui comprenait alors dix spécialités. L'institution vietnamienne concernée est le Collège des technologies (Coltech) à Hanoi. De nombreuses relations existaient entre ces entités avec, notamment, des collaborations de recherche, un master 1+1 (une année en France et une année au Vietnam) en micro et nano technologies et un programme DUO d'échange d'enseignants.

Le Coltech, en tenant compte de ses propres formations et des compétences de ses enseignants, a retenu trois spécialités recherche du master IST : Réseaux et télécoms, Composants et dispositifs électroniques pour les télécommunications, Micro et nanotechnologies (M2).

\section{LANCEMENT DU PROJET}

Le lancement du master IST délocalisé à Hanoï a eu lieu à la rentrée 2006-2007. Parallèlement, en septembre 2006, un bureau du PUF a été créé. Son rôle est 
administratif. Il gère les budgets et organise les missions. Le rôle des partenaires est alors défini : l'université Paris-Sud 11 délivre le diplôme, envoie des missionnaires, fournit le contenu pédagogique de la formation, le Coltech aide à la réalisation du projet, fournit des enseignants et l'intendance (secrétariat et salles) et le PUF supervise l'échange, cela en vue de créer un diplôme délocalisé.

Le coût de la formation a été fixé par l'ambassade de France au Vietnam afin d'être uniforme sur tous les diplômes proposés dans le cadre des PUF. Il est de $2500 \$$ par étudiant et par an. C'est une somme considérable dans un pays où le revenu d'un enseignantchercheur se compte en centaines de dollars par mois (environ $300 \$$ par mois).

Le programme de la première année du master (M1) a été organisé afin de proposer des unités d'enseignement (UE) adaptées aux M2 sélectionnés. Ainsi la formation proposée à Hanoi couvre en M1 un spectre moins large que celui offert en France et le catalogue des UE à choisir en M1 et M2 est très réduit.

\subsection{Achat du matériel}

Les accords pour le prêt des locaux ont été négociés entre le PUF et le Coltech. Il restait à équiper les salles de travaux pratiques (TP) afin de pouvoir dupliquer les expérimentations effectuées par les étudiants en France. En effet, le matériel de TP disponible au Coltech ne répond pas aux besoins (en particulier en électronique).

Cette étape a été longue et délicate. Initialement les achats devaient être faits au Vietnam. Les devis obtenus sur place étaient plus chers que l'équivalent en France et pour du matériel offrant moins de possibilités. Il a donc fallu négocier l'autorisation d'effectuer l'achat en France. Ensuite, des tractations diplomatiques entre l'ambassade de France au Vietnam et le gouvernement vietnamien, liées au paiement des taxes, ont augmenté les délais d'approvisionnement. Finalement, les $29 \mathrm{k} €$ HT (frais de port compris) d'oscilloscopes, alimentations, multimètres, GBF et connectiques diverses sont arrivés en décembre 2008. Il a donc fallu aviser avec le matériel local la première année et certains enseignants français sont venus avec leur matériel afin d'illustrer leur enseignement et réaliser des TP.

Le même genre de problèmes a été rencontré pour l'achat des documents pédagogiques. Peu de compagnies ont le droit d'importer des livres au Vietnam. Il a fallu passer par les librairies autorisées pour les achats initiaux. La complexité et la lenteur de la procédure nous ont conduits à importer les livres par le biais des valises des missionnaires.

\subsection{Formation de formateurs}

Nous avons choisi de créer des binômes francovietnamiens pour assurer l'enseignement des UE. Les deux enseignants sont appelés " correspondants " pour traduire le fait que l'on attend d'eux des échanges suivis. La procédure adoptée pour la gestion des enseignements présente deux intérêts. Le premier en termes de formation de formateurs, et le second en termes de pérennisation du programme. Afin de clarifier le rôle du correspondant vietnamien aux yeux de tous les intervenants, le texte suivant a été rédigé : "Le correspondant doit assister à tous les cours, y compris et surtout aux TD et TP. Il prend une part active aux cours. Ce n'est pas l'assistant de l'enseignant français mais le deuxième enseignant d'un cours à deux professeurs. Il doit compléter l'information donnée par le missionnaire, et adapter le message aux étudiants vietnamiens. Il supervise autant que le missionnaire les $T D$ et TP. Il doit intervenir s'il voit que les étudiants n'ont pas compris. Il doit se procurer tout le matériel pédagogique afin de pouvoir dispenser le même cours l'année suivante. C'est l'interlocuteur privilégié $d u$ missionnaire à tout point de vue et, en particulier, pour d'éventuelles collaborations recherche. " $\mathrm{Au}$ fur et à mesure des promotions et en fonction des binômes, certains sont parvenus à se partager les interventions en cours.

Un autre volet de la formation de formateurs touche aux aspects recherche. 1) Des collègues vietnamiens ont eu des séjours de un à trois mois en France pris en charge par le ministère vietnamien de l'éducation (MOET) pour être accueillis dans des laboratoires de l'université Paris-Sud 11 et prendre part aux enseignements à Orsay. Il y a eu trois missions de ce type en 2007-2008. 2) Les stages de M2 se déroulent, dans la mesure du possible, en co-encadrement avec un tuteur français et un vietnamien. 3) Certains de ces stages donne lieu à des thèses en cotutelle. 4) Les doctorants issus de ces thèses en cotutelle devraient, par la suite, être recrutés au Coltech. 5) Enfin, lors des missions d'enseignement, quelques collègues d'Orsay ont pris des contacts et entament des collaborations de recherche avec des collègues d'Hanoï.

\subsection{Coopération}

La coopération s'établit sur une relation de confiance. L'idée est de faire partager notre pédagogie à nos collègues vietnamiens. En effet, leur enseignement est encore principalement basé sur des cours magistraux. C'est pourquoi, ils sont très intéressés par le déroulement des travaux dirigés (TD), au cours desquels l'étudiant est actif, et des TP, au cours desquels les qualités expérimentales sont développées et les interactions avec les étudiants sont fortes.

On cherche à donner à tous les étudiants la possibilité de profiter au maximum de la présence des deux enseignants (français et vietnamien) lors des cours. Ce qui ressort dans la perception des étudiants vietnamiens de notre enseignement est la forte complémentarité entre cours magistraux, TD et TP. Entre autres, ils sont surpris par la forte implication qu'on leur demande durant les séances. Ils énoncent le fait qu'on leur demande " comprendre le cours pendant le cours » alors qu'ils avaient plus l'habitude de travailler rétrospectivement à l'aide de leurs notes de cours et de livres de référence.

Indépendamment, l'orchestration de cette coopération requiert un fort investissement de la part des res- 
ponsables à Orsay du projet. Tous les ans, des missions de recrutement, jurys, coordinations... sont nécessaires. En outre, il faut une grande réactivité face aux demandes, l'éloignement ralentissant de manière notable les transmissions d'information.

\section{FLUX ET QUALITE}

Le premier recrutement a eu lieu en septembre 2006. Dans cette partie, nous donnons un aperçu des effectifs et des résultats associés.

\subsection{Année 2006-2007}

27 candidats ont été auditionnés pour le M1, 21 ont été acceptés mais seuls 17 se sont finalement inscrits. Sur les 17 candidats de M1, 14 envisageaient comme spécialité de M2 Réseaux et télécoms. Les 3 autres voulaient suivre la spécialité Composants et dispositifs électroniques pour les télécommunications. En cours d'année, il y a eu 5 abandons.

À l'issue de la deuxième session, il y a eu 2 redoublements et 9 passages en M2 (certains avec une UE de M1 à repasser).

On notera que pour cette première année, le recrutement a souffert du manque de notoriété au Vietnam du diplôme. Le manque de recul sur l'intérêt de cette formation très coûteuse pour un Vietnamien et le mauvais affichage des pré-requis ont conduit à des candidatures assez mal ciblées. Le niveau d'anglais des candidats était insuffisant pour suivre les cours dans de bonnes conditions. En outre, cette promotion a subi les aléas du démarrage (planning, matériel de $\mathrm{TP}$, rodage des enseignants...). Une erreur particulièrement flagrante a été la suivante : nous avions autorisé à ce que les cours effectués par des enseignants vietnamiens soient donnés en vietnamien alors que les examens ont été donnés en anglais. Tous les sujets d'examen émanaient d'enseignants du master IST (y compris quand les UE étaient enseignées exclusivement par des collègues vietnamiens), avec un barème de notation qui est celui pratiqué en France. Cela a été très pénalisant pour les étudiants.

Pour compléter les informations concernant cette première promotion, il faut préciser qu'un étudiant de M1 qui avait validé son année ne s'est jamais inscrit en M2. Et que sur les 3 étudiants qui voulaient faire la spécialité minoritaire, les 2 qui avaient validé leur M1 se sont vus financer une année d'études à Orsay pour y suivre la spécialité du M2 IST qu'ils avaient choisie.

Précisons, malgré ce constat, que nous avons fait le choix de maintenir un enseignement et une évaluation équivalents à ceux en France. Nous avons choisi de maintenir le niveau du diplôme.

Suite à cette première année, nous avons instauré une période de remise à niveau d'un mois avant l'entrée en M1. Elle contient des enseignements d'anglais, de mathématiques et d'électronique et est assurée par des collègues vietnamiens.

\subsection{Année 2007-2008}

31 candidats ont été auditionnés, 21 ont été acceptés pour le M1 et 2 directement en M2 (il s'agit d'étudiants âgés de 34 et 36 ans suivant le comptage français ${ }^{1}$, qui parlent très bien anglais, n'ont pas pour objectif de faire de thèse mais veulent simplement progresser dans leur carrière en suivant les cours).

En M1, seuls 17 se sont inscrits. L'effectif du M1 a donc été de 19 étudiants avec les 2 redoublants. Il y a eu 5 abandons en cours d'année. 8 nouveaux étudiants ont validé leur M1 en première session, les étudiants de M2 qui avaient des UE à repasser les ont validées.

En M2 Réseaux et télécoms, sur les 9 inscrits (7 viennent du M1, et 2 ont été recrutés directement en M2), il y a eu 2 abandons et 5 étudiants ont validé leur année à l'issue de la première session.

Les 2 étudiants qui sont venus en France suivre leur M2 ont obtenu le master, surmontant de nombreuses difficultés, notamment liées à une mauvaise maîtrise de la langue française.

L'année de M1 2007-2008 a été une réussite. Nous avons su tirer profit de l'expérience de l'année antérieure. Certains intervenants ont été remplacés, et nous avons aussi mis en place certains cours par visioconférence.

Le M2 Réseaux et télécoms a connu les difficultés liées à sa mise en place (organisation des TP, nouveaux intervenants, et problème d'encadrement des stages recherche).

\subsection{Année 2008-2009}

37 candidats ont été auditionnés (aucun n'a pu être recruté directement en M2). 24 ont été retenus. Les auditions ont été faites en deux temps. 18 candidats ont été retenus en juillet, et 6 en septembre.

Sur les 18 candidats retenus en juillet, seuls 11 s'étaient inscrits en septembre alors que le mois de remise à niveau commençait. Ce fort désistement est dû à une tentative de lutte contre l'abandon en cours d'année. Pour éviter que les étudiants paient le premier semestre avant de se rendre compte que le rythme est trop difficile pour eux, nous avons organisé des discussions ouvertes entre anciens et nouveaux étudiants de M1 afin que l'information circule. La suite permettra d'évaluer la réussite de cette procédure.

L'année 2008-2009 est aussi l'année d'ouverture du M2 Micro et nanotechnologies. L'ouverture résulte d'une volonté forte de l'université Paris-Sud 11 ainsi que du Coltech de former des étudiants dans cette spécialité au Vietnam. Les étudiants recrutés (10 sur 11 auditionnés) ne sont pas passés par le M1 du PUF mais font partie des meilleurs étudiants en micro et nanotechnologies au Vietnam. Tous avaient déjà été recrutés par des laboratoires du domaine pour assister un professeur dans son activité de recherche.

\subsection{Généralités}

Sur les effectifs mentionnés ci-dessus, la proportion d'étudiants salariés a toujours été supérieure à $50 \%$ (même si ce taux diminue tous les ans). Cela nous oblige à organiser la formation en cours du soir. Il est pos-

${ }^{1}$ Au Vietnam, la période fœtale est comptée, ils ont donc 35 et 37 ans. 
sible que le coût de la formation soit en cause dans cette " sélection » d'étudiants. Les cours ont donc lieu de $18 \mathrm{~h}$ à $21 \mathrm{~h}$ en semaine et le week-end.

Des bourses sont accordées tous les ans au mérite. Pour l'année 2006-2007, ces bourses n'ont concerné que le M1. Le nombre de bourses ainsi que le pourcentage qu'elles représentent par rapport aux frais d'inscription est : 3 bourses couvrant la totalité des frais d'inscription, $175 \%, 250 \%$ et $425 \%$. C'est-à-dire que tous les étudiants admis en M2 (même s'ils n'avaient pas validé totalement leur M1) ont obtenu une bourse. Ces bourses viennent du PUF, qui reverse une partie des frais d'inscription, du Coltech qui a un budget propre pour ce programme, ou d'organismes extérieurs (compagnies, fondations...). En 2007-2008, pour le M1, 6 bourses de $100 \%$ et, pour le M2, 3 de $100 \%$ ont déjà été distribuées, d'autres sont à venir.

La langue d'enseignement est l'anglais du fait du trop faible nombre d'étudiants vietnamiens maîtrisant le français. Les formations du PUF qui ciblaient les étudiants francophones n'ont du reste pas été ouvertes, faute d'un effectif suffisant. Néanmoins, il nous semblait important de privilégier la communication sur la défense de la francophonie et, au cours du master, les étudiants ont 300 heures d'enseignement de français.

\section{INTÉRÊTS DU PROJET}

L'intérêt majeur de ce projet est de créer des relations entre enseignants et chercheurs. Le fait de constituer des équipes mixtes pour toutes les étapes du processus (sélection, déroulement, contrôle des connaissances...) permet, lorsqu'un dialogue franc est possible au sein de l'équipe, de se poser des questions sur nos habitudes ou nos techniques et de progresser. Ensuite, chaque intervenant trouve différents avantages dans ce programme.

\subsection{Université Paris-Sud 11}

L'UFR des sciences a des retombées directes de ce programme :

- le PUF verse $2500 €$ par classe et par an pour le master IST,

- les étudiants sont inscrits à l'université Paris-Sud 11,

- les étudiants réalisent des stages de M2 soit en France soit en co-encadrement,

- de nouveaux contacts industriels se mettent en place,

- des contacts recherche émanent des missionnaires,

- un flux de doctorants issus du M2 arrive dans les laboratoires d'Orsay.

\subsection{Coltech}

Le Coltech reçoit un budget du MOET en contrepartie de l'accueil de ce programme. Ce budget est en partie reversé aux étudiants sous forme de bourses mais sert aussi à envoyer des enseignants-chercheurs ou des techniciens en France pour une durée de 1 à 3 mois pour des missions recherche ou formation.Des salles de TP ont été équipées au cours de ce programme.

\subsection{Enseignants français}

Les missionnaires français sont des enseignantschercheurs ou des chercheurs CNRS, en provenance des campus de l'université Paris-Sud 11, de l'ENS Cachan, mais aussi de Supélec partenaire du master IST pour quelques spécialités de M2. Ils ne s'occupent pas des aspects administratifs de leur voyage: le PUF achète le billet d'avion (souvent en catégorie intermédiaire), à l'arrivée leur visa est prêt, et un chauffeur les conduit de l'aéroport à leur hôtel. Une somme forfaitaire donnée à l'arrivée en liquide couvre les frais de séjour (per diem), les heures d'enseignement sont rémunérées au tarif français $(60 € / \mathrm{h})$ par le PUF.

Les retours des enseignants français sont particulièrement positifs, tous les missionnaires ont été reconduits et n'envisagent pas de ne pas renouveler l'expérience dans l'immédiat. Ils mettent en avant l'aspect bénéfique pour leur enseignement, en favorisant une réflexion de fond sur la nature de leur cours du point pédagogique.

\subsection{Enseignants vietnamiens}

Quand le correspondant assiste aux cours dispensés par un missionnaire, il est rémunéré par le Coltech comme s'il assurait le cours lui-même. Quand il enseigne pour le PUF, le tarif horaire d'enseignement (20 \$/h de cours) est largement supérieur au tarif en vigueur au Vietnam. C'est aussi une reconnaissance d'avoir été proposé par sa hiérarchie pour intervenir dans ce programme.

\section{5. Étudiants}

Ce programme jouit d'une attention particulière de la part de l'ambassade de France au Vietnam et du MOET. Les étudiants des promotions passées (pour ceux qui ont réussi) ont pu bénéficier d'avantages particuliers : séjours en France payés, bourses... Ils travaillent dans des conditions appréciables : équipements récents, salles climatisées, ordinateurs à disposition, bibliothèque dédiée ... Il y a de fortes raisons de croire que leur avenir post-diplôme est assuré.

\section{DIFFICULTES}

Il semble important de signaler un certain nombre de difficultés. Tout d'abord, il a fallu du temps pour que le rôle de chacun se dégage clairement. Les différents partenaires attendant beaucoup les uns des autres sans savoir exactement qui tirait profit de cet échange. Cela est renforcé par le nombre d'interlocuteurs du programme. Une autre difficulté est liée à la perception que l'on peut avoir de la notion de missionnaire. Il a fallu au minimum une année pour que les missionnaires parviennent à établir une relation avec le correspondant montrant qu'ils ne considéraient pas être les seuls à savoir transmettre des connaissances. L'intervention des enseignants français est imposée par le PUF et demandée par les étudiants.

Nous devons aussi évoquer les différences de fonctionnement entre les deux pays impliqués. Les protocoles à respecter au Vietnam ne sont pas les mêmes qu'en France et il a fallu les découvrir. Il est important au Vietnam que les contrats des enseignants soient précis, 
que toutes les interventions soient rémunérées, que les salles soient réservées auprès de tous les responsables. La communication entre collègues ne repose pas sur les mêmes codes en France et au Vietnam : par exemple, le respect dû à l'âge impose plus de retenue au Vietnam.

Enfin, nous avons rencontré deux difficultés relativement anecdotiques :

- il a fallu démontrer la qualité du programme car les cours du soir ont très mauvaise presse,

- les sonorités et orthographes des noms vietnamiens demandent une concentration particulière dans la réalisation des jurys.

\section{CONCLUSION ET PERSPECTIVES}

Cette expérience est très enrichissante, vivante et agréable. Elle repose en partie, du côté vietnamien, sur la volonté de mieux connaître la France. En particulier, les étudiants vietnamiens veulent suivre des cours donnés par des enseignants français. Cela n'est pas particulièrement de très bon augure pour la pérennisation du programme.

Nous n'avons pas encore eu la possibilité d'envoyer des étudiants français suivre le master à Hanoï mais cela reste d'actualité.

Année après année, le programme s'améliore grâce notamment à l'augmentation du niveau de confiance entre intervenants et à la crédibilité acquise auprès des étudiants. Des tentatives sont faites tous les ans pour ne pas renouveler les erreurs des années antérieures (utilisation de la visioconférence, mesures pour éviter l'abandon...).

Un volet recherche sera progressivement mis en place avec la constitution d'équipes mixtes développant des projets de recherche communs.

Les auteurs remercient particulièrement Cécile Durieu en tant que coresponsable du master IST pour l'ENS Cachan pour son aide inestimable dans le bon fonctionnement du master IST délocalisé à Hanoï. 